\title{
AIR GAP CONTROL AT SENSOR HEAD OF MOBILE MANIPULATOR ON MINE DETECTION ROBOT
}

\author{
Yoshitomo ASAI ${ }^{*}$ and Kenzo NONAMI ${ }^{* *}$ \\ * Graduate School of Science and Technology, Chiba University, asai@mec2.tm.chiba-u.ac.jp \\ **Department of Electronics and Mechanical Engineering, Chiba University, nonami@meneth.tm.chiba-u.ac.jp
}

\begin{abstract}
In this paper, we propose the on-line adaptive trajectory planning by evaluating acceleration of designed trajectory. The tracking speed should be changed with curvature of the given trajectory. In order to change its speed, the time parameter is scaled by designed trajectory acceleration. We verify that the control performance is maintained even if a drastic change of reference is given by means of changing time parameter.
\end{abstract}

\section{INTRODUCTION}

Mine detection and clearance in mine buried countries has become an issue of international emergency. The such mine varied countries have been developing, however it is necessary for them to have humanitarian support from Japan, US and western countries. This fact is born especially apparent during the Afghanistan reconstruction support conference held in Tokyo in January, 2002. There are 110,000,000 and more mines in over 70 countries such as Egypt, Iran, Angola and Afghanistan. (Report from United Nations Mine Clearance Data Base)

In such conditions, we developed mine detection six-legged walking robot named COMET and we have proposed COMET as the core technology of the humanitarian mine detection for such countries. As a concrete method of mine detection, we consider an available approach by means of a high sensitivity mixed sensor based on a radar and a metal sensor, which is attached at the tip of manipulator installed in front of COMET.

The performance of detecting mines is highly sensitive to the distance between the ground and the sensor (air gap). The accuracy decreases by improper air gap and mines might be undetectable. This air gap fluctuates according to the steps of the robot or the nature of the terrain. Just following the designed trajectory as well causes fluctuation in the air gap because system can not fully follow it. On the other hand, a change in posture insignificantly affects the change in the air gap because COMET is does not mo-

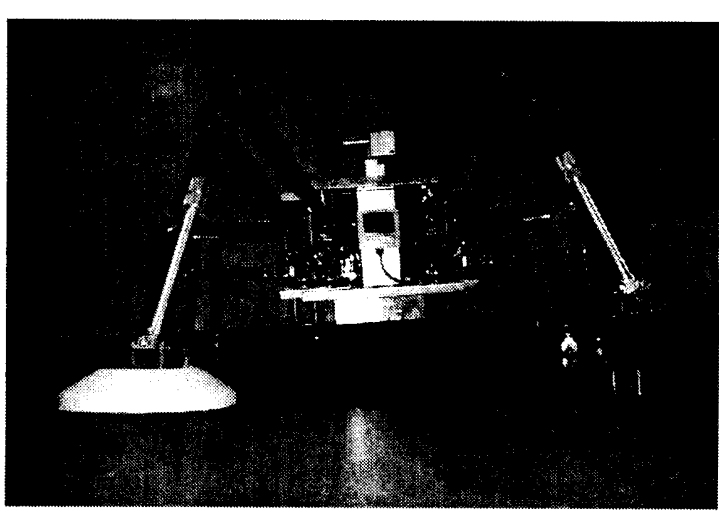

Fig. 1 Overview of COMET-II

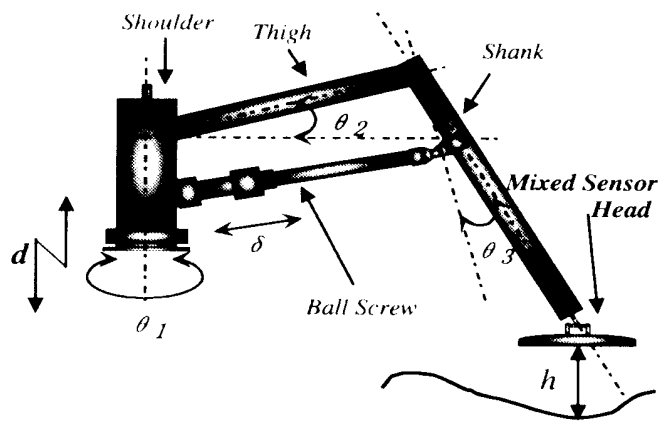

Fig.2 Configration of manipulator and air gap control

Table 1 Spec. of manipulator

\begin{tabular}{|c|c|}
\hline & spec. \\
\hline Shoulder & $220[\mathrm{~mm}]$ \\
\hline Thigh & $670[\mathrm{~mm}]$ \\
\hline Shank & $690[\mathrm{~mm}]$ \\
\hline \hline & range \\
\hline$\theta 1$ & $-70 \sim 90[\mathrm{deg}]$ \\
\hline$\theta 2$ & $-20 \sim 45[\mathrm{deg}]$ \\
\hline$\delta$ & $-100 \sim 160[\mathrm{~mm}]$ \\
\hline
\end{tabular}

ve when he detects mines. An on-line trajectory planning that continuously adjusts accordingly to uneven terrain is needed to maintain the proper air gap. In order to stabilize air gap fluctuations, this study aims to create on-line 
adaptive trajectory planning by evaluating acceleration of designed trajectory and a time scale parameter derives to keep a constant air gap against uneven terrain.

\section{CONTROLLED OBJECT}

The controlled objects are two manipulators on mine detection robot COMET-II as shown in Fig.1. That model is shown in Fig. 2 and its specification is shown in Table 1. Where the mine detection sensor is attached at the top of the left manipulator in front side, an ultrasonic sensor for measuring air gap is equipped at the tip of the other. These manipulators have three-degree-of freedom, and a DC motor is installed in each joint. The host computer supplies the control voltage which is converted to the control current to the TITEC DRIVER. Furthermore, each joint angle is measured by the potentiometer installed on each joint.

\section{MODELING}

Modeling was done for each joint which mean the second order system as shown in Eq.(1) via frequency responses, step responses and the following responses. A change in the form due to the operation of each joint was ignored. The results of experiments and a simulation of frequency response and the step response of shoulder are shown in Fig. 3 and 4 , respectively.

$$
P=\frac{\alpha \omega_{n}^{2}}{s^{2}+2 \zeta \omega_{n} s+\omega_{n}^{2}}
$$

\section{ON-LINE ADAPTIVE TRAJECTORY PLANNING}

Trajectory planning is necessary for good performance. In order to detect mines that responded rough terrain, it is necessary to make adaptive trajectories considered characteristics of the system. Abe and Tsuchiva ${ }^{[2]}$ suggested trajectory planning in two link manipulator by means of mapping path to the joint space. It is approximated by an arc, and its frequency make into cut-off frequency when that differ from cut-off frequency. However it is complicated on three or more degrees of freedom. Although Matsushita ${ }^{[3]}$ applies control system configuration for trajectory planning problem and obtained good responses, when the control system is constructed by using his method of approximation, an approximate error increases where the change in the rapid desired value is dramatic such a step response. And it is further pointed out that the control becomes unstable with deterioration. Ozaki ${ }^{[4]}$ proposes an off-line trajectory planning method that estimates joint trajectory value of the evaluation function small, under the terms of restraint. Furthermore, Yamamoto ${ }^{[5]}$ obtains effective results by the evaluation function using B-spline curve. These techniques, however, are different from the proposed method in the point of off-line trajectory planning.

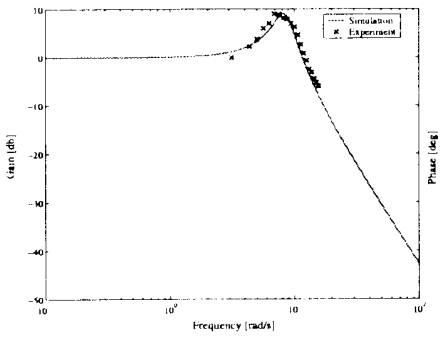

(a) Gain

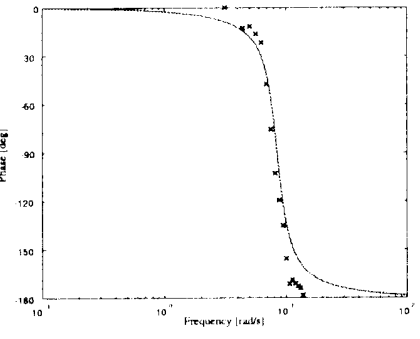

(b) Phase
Fig.3 Frequency response of shoulder
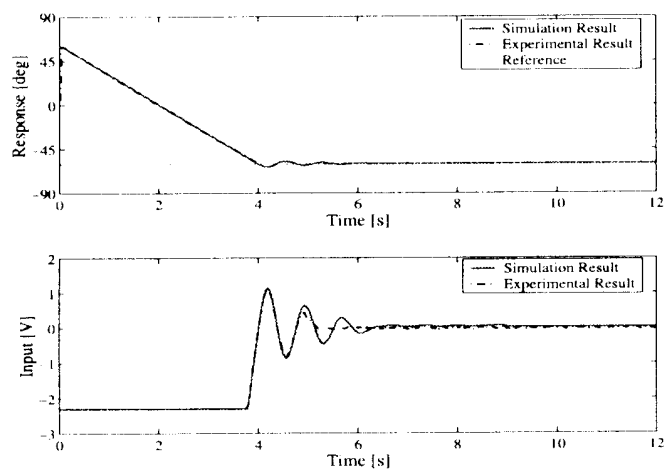

Fig.4 Step response of shoulder

\subsection{On-line adaptive trajectory planning}

In this section, the smooth trajectory is obtained against rapid change of trajectory using future and past information of path.

First, the air gap is measured in future step for mine sensor by the ultrasonic sensor attached to the tip of the left manipulator in foreside as shown in Fig.1. The coordinate is attached on each manipulator, We define the vertical upper direction is set as the $z$ coordinate, the proceeding direction of the robot is the $y$ coordinate, and the cartesian space is assumed as the $x$ direction. Designed trajectory in the $x$ direction and the $y$ direction are calculated by given equations. And then designed trajectory in $z$ direction are set by means of measured air gap, which is mapped into the joint space. Next, designed trajectory acceleration of each joint, $\ddot{\theta}_{j}(j=1,2,3)$, are calculated, and count weighted acceleration average $a_{j}$ by Eq.(2) and (3),

$$
a_{j}=\sum_{i=1}^{p} \alpha_{j}(i)|\ddot{\theta}(k+i)|+\beta_{j}|\ddot{\theta}(k)|+\sum_{i=1}^{q} \gamma_{j}(i)|\ddot{\theta}(k-i)|
$$

where $p, q$ are a number of preview step and pastview step respectably.

$$
\alpha_{j}(i+1)<\alpha_{j}(i)<\beta_{j} \quad, \quad \gamma_{j}(i+1)<\gamma_{j}(i)<\beta_{j}
$$

After that, $a_{j}$ is mapped $T_{d}$ using mapping function as shown in Fig. 5 and Eq.(4). This mapped value will be 
referred to as intensity of slowdown requirement(ISR).

$$
T_{d_{j}}=\eta_{j}\left(\frac{a_{j}}{a_{j}+\varepsilon_{j}}\right) \quad\left(0<\eta_{j} \leq 1,0<\varepsilon_{j}\right)
$$

where, $\eta_{j}, \varepsilon$ are the designed parameters.

Then the largest value of ISR which is calculated independingly in each joint is set for the ISR at the present. and a time parameter $T_{k}$ is up dated from Eq.(5).

$$
\begin{aligned}
T_{d} & =\max \left\{T_{d_{1}}, T_{d_{2}}, T_{d_{3}}\right\} \\
T(k+1) & =T(k)+1-T_{d}
\end{aligned}
$$

This method can generate ISR at present step by Eq.(5) against a change of designed trajectory in the past and future steps by means of adopting Eq.(2). Then, the nonlinearity of the desired trajectories are considered as small as ignored in minute time, and the on-line adaptive trajectory planning is done by interpolating a desired value with a linear form based on Eq.(6).

$$
\tilde{R}_{j}(k)=T_{d} \cdot R_{j}(k)+\left(1-T_{d}\right) \cdot R_{j}(k+1), \quad(j=1,2,3)
$$

where, $\tilde{R}_{j}(k)$ are values which are given to system, $R_{j}(k)$ and $R_{j}(k+1)$ are designed trajectory in present step and next step respectably

\subsection{Taking into account of limit angular velocity}

It is necessary that the trajectory planning with consideration of limit angular velocity by the influence of nonlinear friction.

At first, the step responses when terms of saturation of the shoulder actuator are changed, are shown in Fig.6. Moreover, results while the actuator of Fig. 6 are saturated, are analyzed by using regression analysis, and that average angular velocities are shown in Fig.7.

Thus when the input to the actuator exceeds $0.4[\mathrm{~V}]$, it is found that the angular velocities saturate regardless of the size of the input. It seems that the restriction of not saturation of the actuator but velocity of the hardware. In the same manner, similar limit angular velocities exist in other joints.

The limit angular velocity is estimated each joint in advance, if each angular velocity value is over limit, Eq.(7) produces ISR $T_{d_{j}}^{\prime}$ so that the angular velocity of the designed trajectory becomes limit angular velocity. The largest value of ISR is selected as ISR at present shown Eq.(8) by comparing all of ISR obtained Eq.(5).

$$
\begin{gathered}
T_{d_{j}}^{\prime}=\kappa_{j}\left(1-\frac{\tilde{\omega}_{j}}{\omega_{j}}\right) \quad\left(0<\kappa_{j} \leq 1, j=1,2,3\right) \\
T_{d}=\max \left\{T_{d_{1}}, T_{d_{2}}, T_{d_{3}}, T_{d_{1}}^{\prime}, T_{d_{2}}^{\prime}, T_{d_{3}}^{\prime}\right\}
\end{gathered}
$$

where, $\kappa_{j}$ is the adjustment weighted value.

And then, the largest ISR is chosen as ISR at present time and trajectory planning is done by interpolating desired trajectories based on Eq.(6).

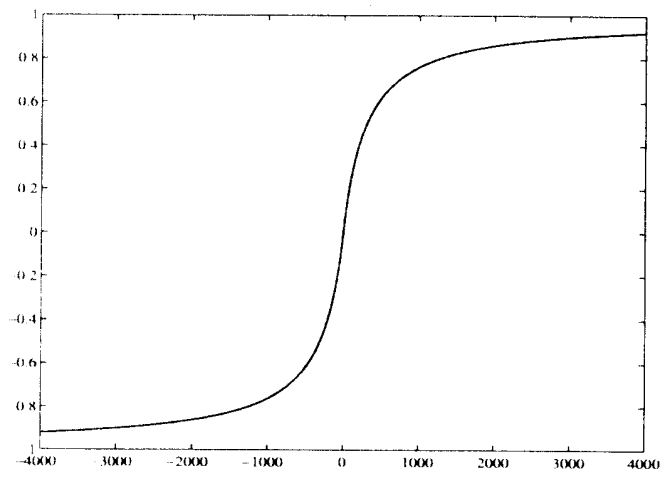

Fig.5 Mapping function
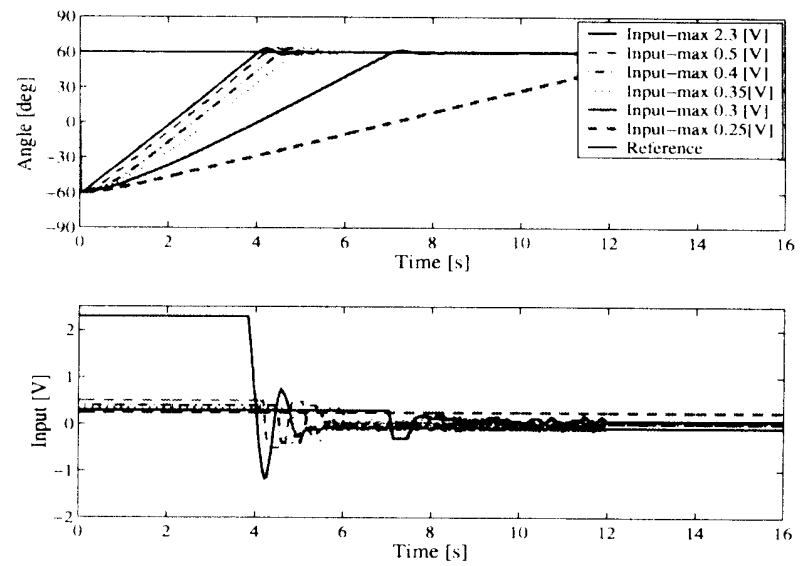

Fig.6 Step responses

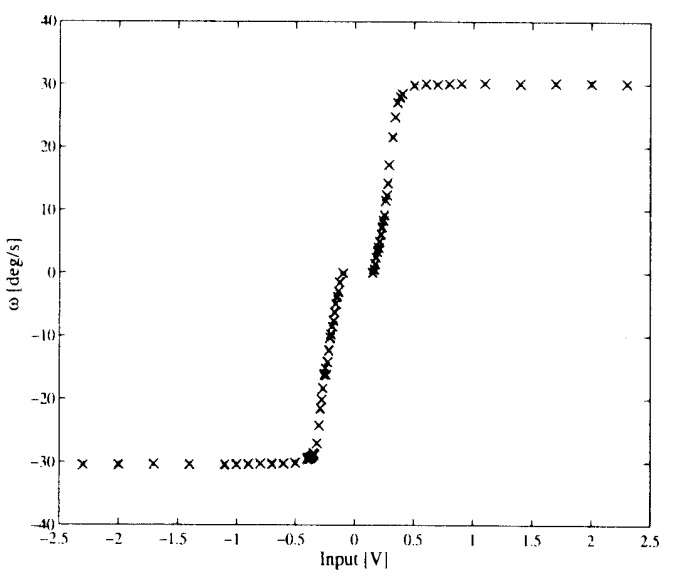

Fig.7 $\omega$ saturation

\subsection{Taking into account of initial response}

ISR is also effective in an initial value response and produces smooth trajectory. First angle velocity is referred to Eq.(9) and Eq.(10), ISR is generated using Eq.(2) to 
Eq.(6) and it improves the following performance to the initial value by changing a time scale parameter when the initial value is different from the initial output of the system.

$$
\begin{gathered}
\dot{\theta}_{j}(1)=\frac{R_{j}(1)-y_{j}(0)}{\Delta t} \quad(j=1,2,3) \\
\ddot{\theta}_{j}(1)=\frac{\dot{\theta}_{j}(1)}{\Delta t} \quad(j=1,2,3)
\end{gathered}
$$

where, $\Delta t$ is the sampling time, $R_{j}(a)$ is the initial value of base reference, $y_{j}(0)$ is the initial output.

\section{SIMULATION AND EXPERIMENT}

The simulation and the following experiments are done to verify the validity of the proposal techniques. These were done only by using the manipulator which an ultrasonic sensor was attached. The designed trajectories are given on a program as sinsoidal wave that its amplitude is $A=0.85$ in the $x$ axis, $y=0.85[\mathrm{~m}]$. And if the value of $x$ satisfies $-0.49[\mathrm{~m}]<x<-0.33[\mathrm{~m}], z=-0.1[\mathrm{~m}]$ is given in the $z$ axis, else $z=-0.27$. Besides, nonlinear friction, saturation of actuators, and limit angular velocity are considered in the simulation.

At first, from Fig. 8 to Fig. 11 and Fig. 21 depict experimental results and simulation results using proposed method, when the period is given as $18[\mathrm{~s}]$ that wave is sinsoidal wave in the $x$ direction as the designed trajectories. And conventional method results are shown in Fig.12 to Fig.15, giving period is $48[\mathrm{~s}]$ that wave is also sinsoidal wave in the $x$ direction

ISR is increased in $T=0[\mathrm{~s}]$ to converge on initial reference in Fig.21. And change of references is smoothed corresponding to ISR. Moreover ISR affected the limit angular velocity of shank is large until $T=25[\mathrm{~s}]$, trajectory is planed corresponding to it. Comparing Fig. 10 with Fig.14, although tracking performance decreases accuracy in Fig.14, good results are obtained by means of on-line adaptive trajectory planning in Fig.10. ISR of thigh and shank adapts to drastic change of references which take place in the future step from $T=25[\mathrm{~s}]$ to $T=40[\mathrm{~s}]$. Furthermore, ISR of limit angular velocity of thigh and shank is generated from $T=30[\mathrm{~s}]$ to $T=35[\mathrm{~s}]$ in Fig.21. Analyzing $T=30[\mathrm{~s}]$ to $T=35[\mathrm{~s}]$ in Fig.9 and Fig.13, they verify the validity of the proposal techniques. Figure 11 and Fig. 15 depict tracks. According to them, the proposed techniques obtain effective results. Moreover, this techniques maintain its performance against a sharp change of reference in Fig.11(a). On the other hand the conventional method could not obtain practical results in Fig.15(a). Furthermore, the system converges to the initial reference in Fig.11.

Next, the results which are added noise of an ultrasonic sensor and potentiometers on references in the $z$ direction is shown in Fig.16 to Fig.18 and Fig.22.

The trajectory planning is done with the proposed method using low-pass filter to reduce a sensitivity to sensing noise. When the subtracted value of angular velocity shown in Eq.(11) satisfy $\delta$, that value passes through the filter. Where $\delta$ is a boundary.

$$
\left|\dot{\theta}_{j}(k)-\dot{\theta}_{j}(k-1)\right|<\delta_{j} \quad(j=1,2,3)
$$

Setting the boundary only effects sensing noise, and maintain the performance to a drastic change of reference. Figure $19(\mathrm{a})$ and (b) show good results although there is sensing noise.

\section{CONCLUSION}

It is proposed that the on-line adaptive trajectory planning by evaluating acceleration of the designed trajectories and changing time scale parameter in order to obtain good performance. Furthermore, these techniques are able to consider that the limit angular velocity caused the limitation of the mine detection speed of the robot. It is also effective when there is noise which is measured the ultrasonic sensor and potentiometers because low-pass filters reduce this noise.

This method does not cause complexity in the case of multi-degree-of freedom, so it will be applied to these manipulators, and reduce the impact when a leg is dropped on ground because of evaluating acceleration in the future steps.

\section{REFERENCES}

[1] Nonami K.,Q.J.Huang: Humanitarian mine detection six-legged walking robot COMET-II with two manipulators, Prof. of the 4th Int. Conf. On Climbing and Walking Robots, pp.989-996, (2001)

[2] Satoshi Abe Takeshi Tsuchiya : Robot Manipulator Path Control Based on Variable Speed Trajectory Planning, Journal of the Robotic Society of Japan, Vol.8, No.1, pp.1-8, (1990)

[3] Akihiko Matsushita Takeshi Tsuchiya : On-line Planning for a Desired Signal and Its Application to Robot Manipulators, Journal of the Robotic Society of Japan, Vol.13, No.8, pp.1160-1168, (1995)

[4] Hiroaki Ozaki Motoji Yamamoto Akira Mohri : Planning of Joint Trajectories of Manipulators with Geometric Path Constraints, Journal of the Society of Instrument and Control Engineers, Vol.23, No.3, pp.288-293, (1987)

[5] Motoji Yamamoto Hiroaki Ozaki Akira Mohri: Planning of Joint Trajectories of Manipulators by Iteratively Improving Method, Journal of the Society of Instrument and Control Engineers, Vol.24, No.9, pp.942-947, (1988) 

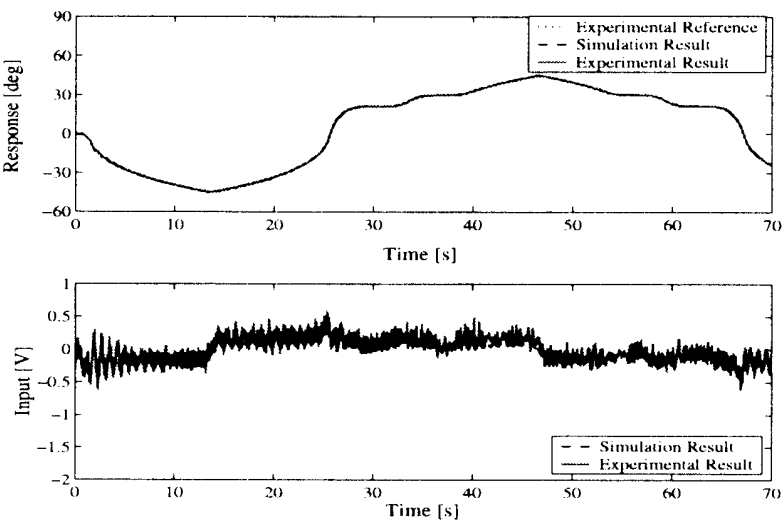

Fig.8 Response of shoulder with adaptive reference
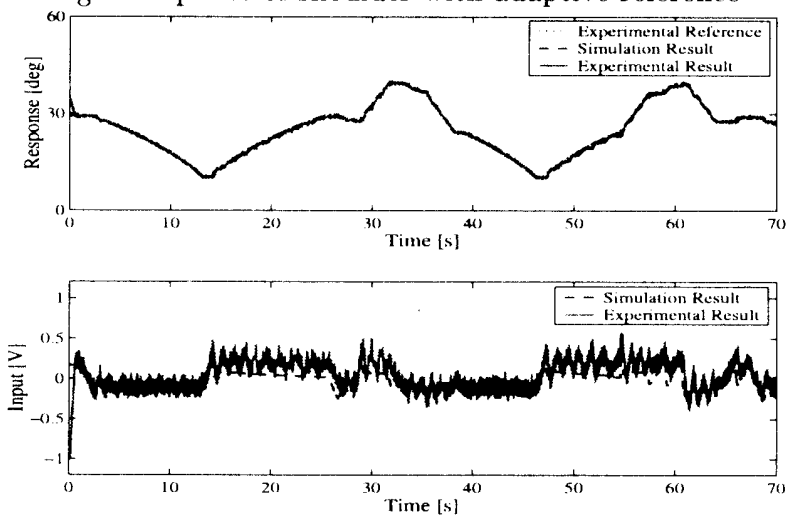

Fig.9 Response of thigh with adaptive reference
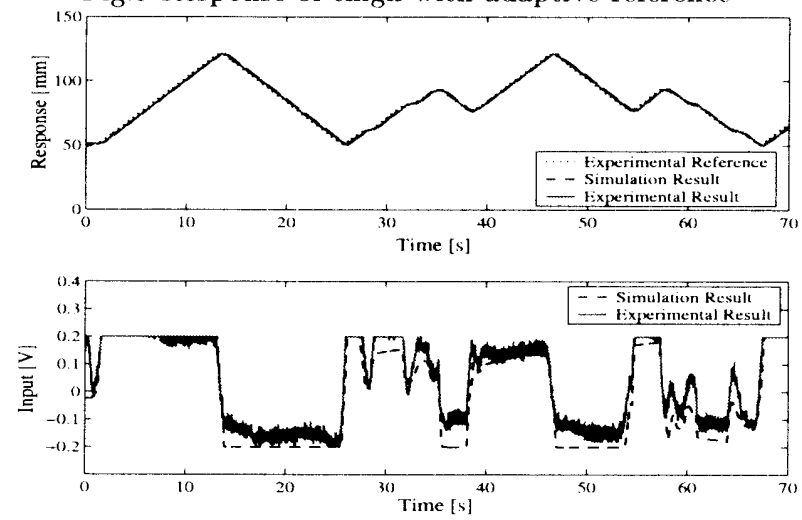

Fig.10 Response of shank with adaptive reference

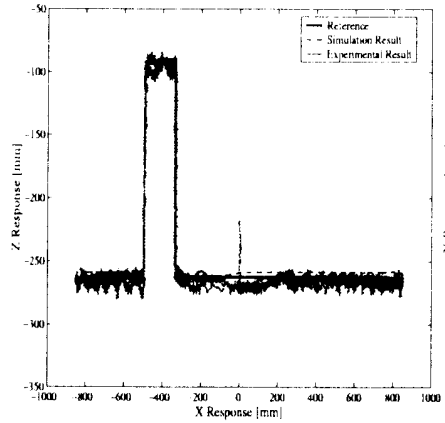

(a) Trajectory in Z-X plane

(b) Trajectory in X-Y plane Fig.11 Trajectory with adaptive reference
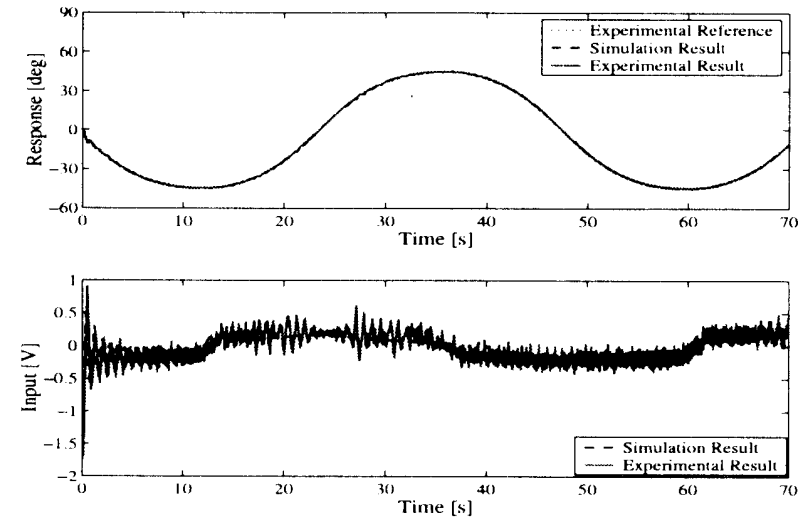

Fig.12 Response of shoulder without adaptive reference
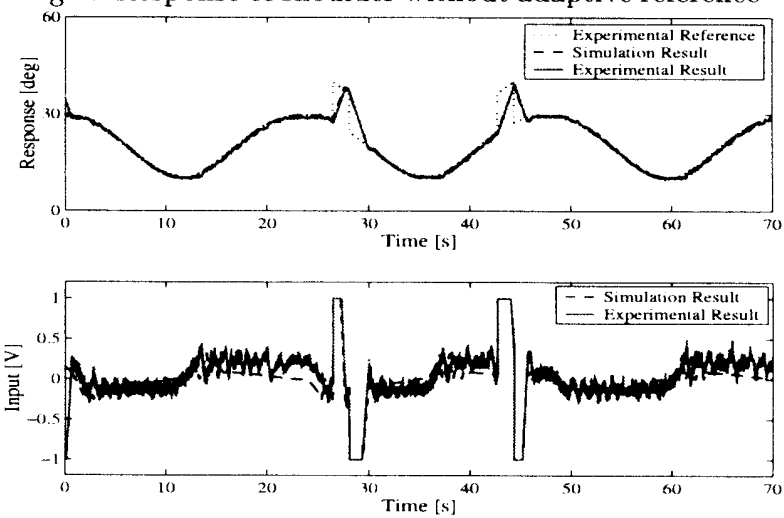

Fig.13 Response of thigh without adaptive reference
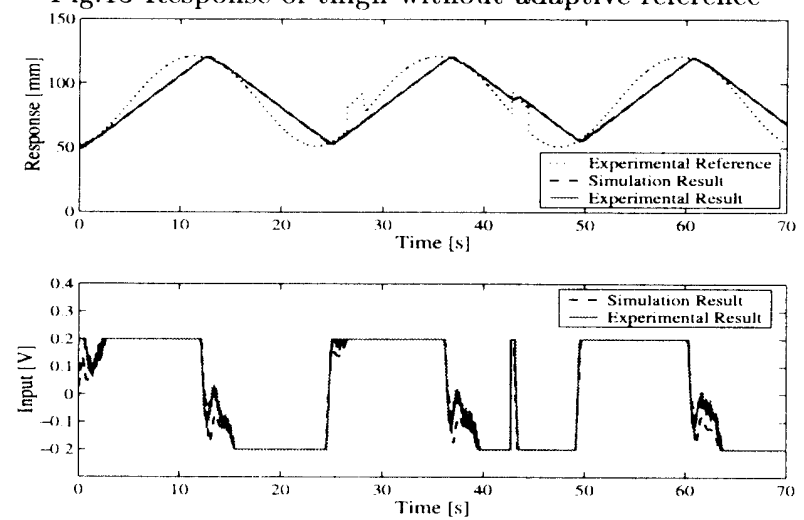

Fig.14 Response of shank without adaptive reference

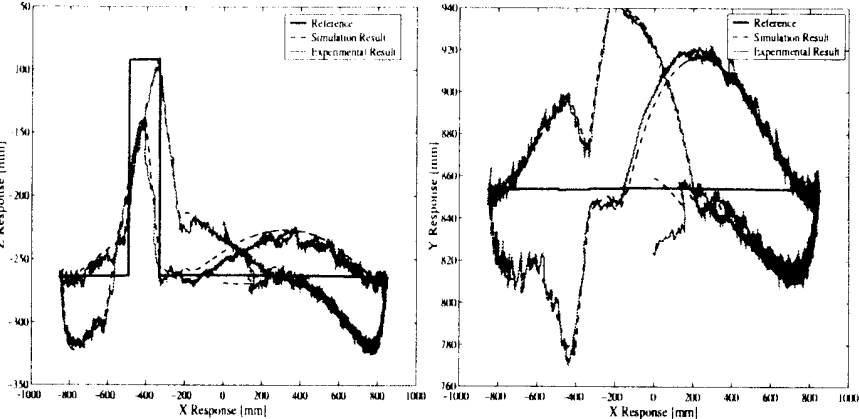

$\begin{array}{ll}\text { (a) Trajectory in Z-X plane } & \text { (b) Trajectory in X-Y plane }\end{array}$ Fig.15 Trajectory without adaptive reference 

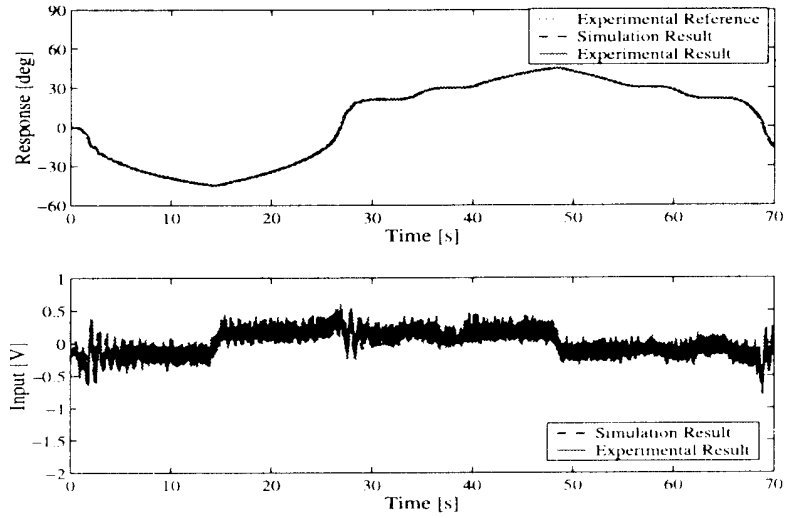

Fig.16 Response of shoulder with ultrasonic sensor
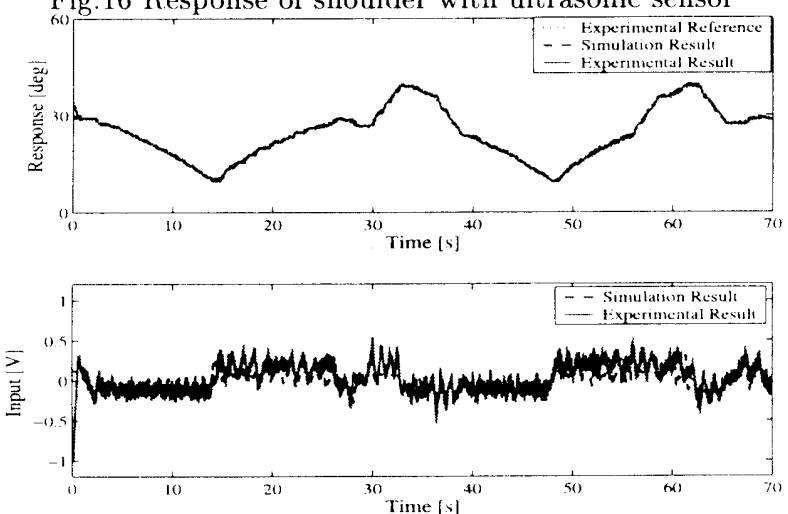

Fig.17 Response of thigh with ultrasonic sensor
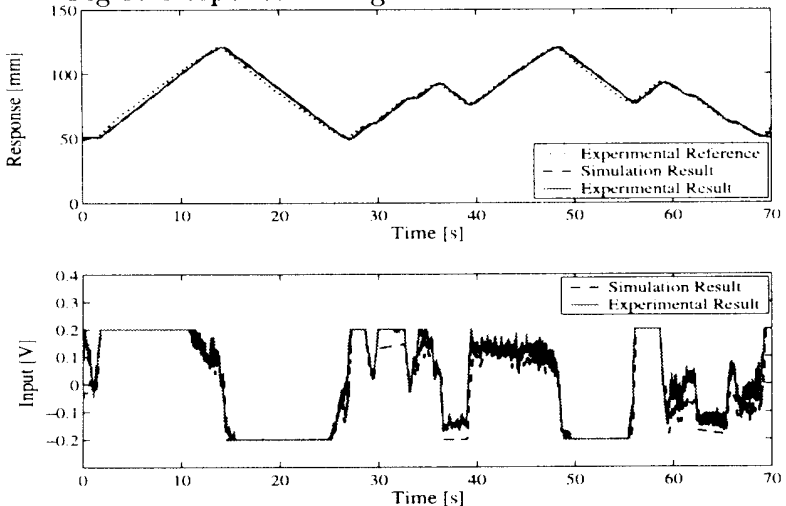

Fig.18 Response of shank with ultrasonic sensor

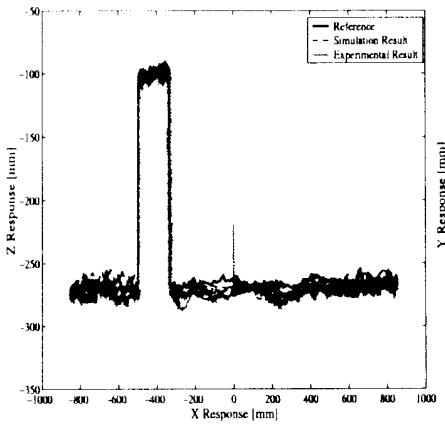

(a) Trajectory in Z-X plane

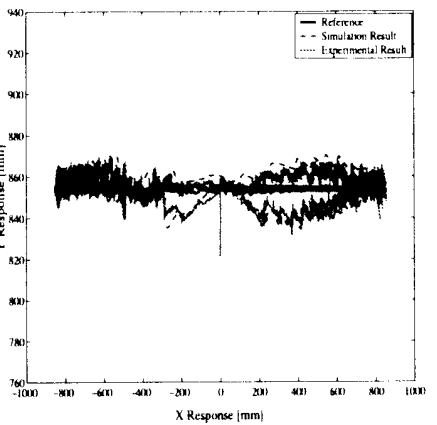

(b) Trajectory in X-Y plane

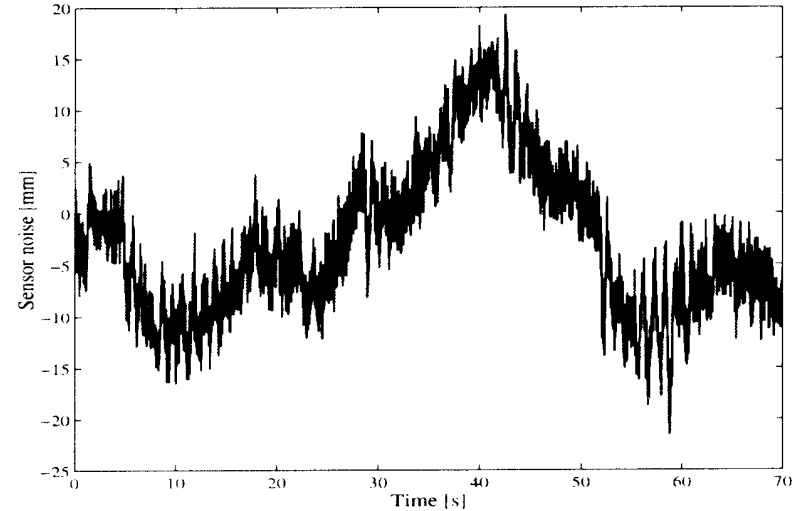

Fig.20 Ultrasonic sensor and potentiometer noise

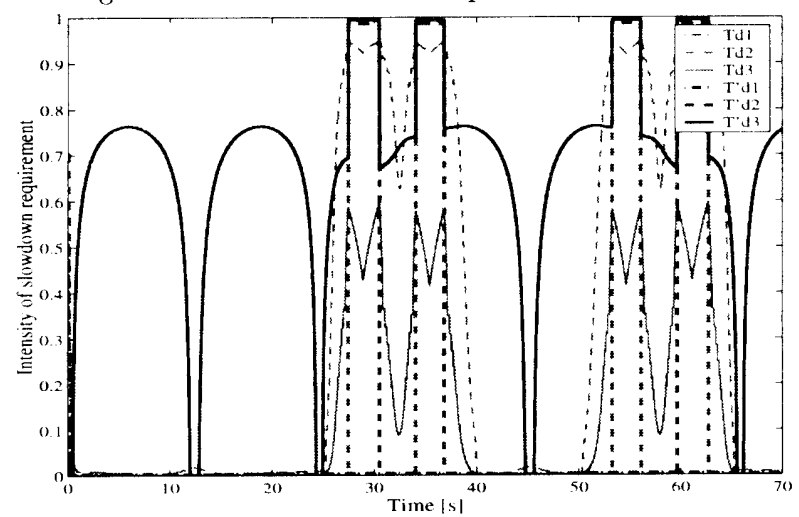

Fig.21 Intensity of slowdown requirement without noise

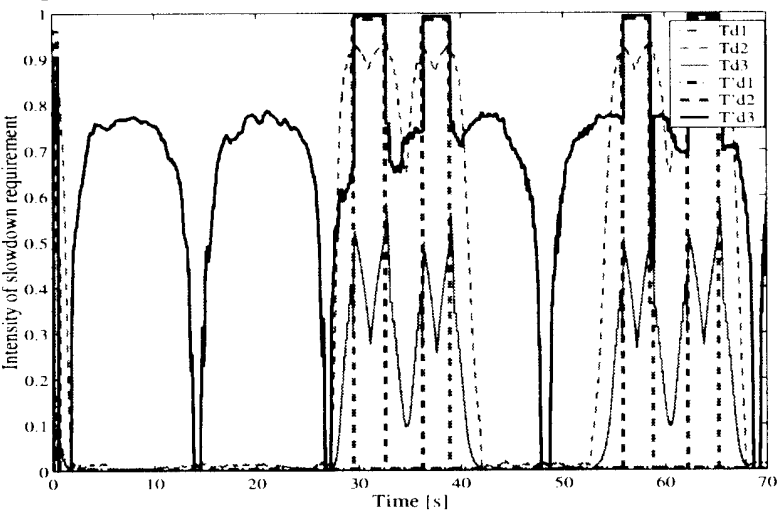

Fig.22 Intensity of slowdown requirement with noise

Fig.19 Trajectory with ultrasonic sensor 